Please do not remove this page

RMIT

UNIVERSITY

\title{
The forced radiation efficiency of finite size flat panels that are excited by incident sound
}

Davy, John

https://researchrepository.rmit.edu.au/esploro/outputs/9921863948601341/filesAndLinks?institution=61RMIT_INST\&index=null

Davy, J. (2009). The forced radiation efficiency of finite size flat panels that are excited by incident sound. Journal of the Acoustical Society of America, 126(2), 694-702. https://doi.org/10.1121/1.3158820

Document Version: Accepted Manuscript

Published Version: https://doi.org/10.1121/1.3158820

Repository homepage: https://researchrepository.rmit.edu.au

(C)2009 Acoustical Society of America

Downloaded On 2023/04/27 00:09:03 +1000

Please do not remove this page 


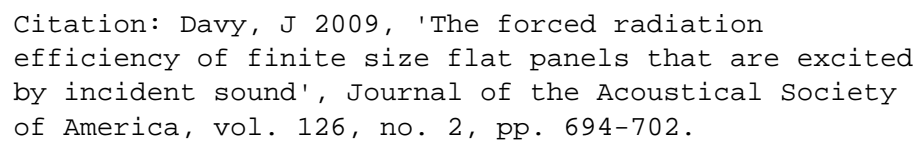

The forced radiation efficiency of finite size flat panels which are excited by incident sound ${ }^{\mathrm{a}}$

John L. Davy ${ }^{\text {bc }}$

School of Applied Sciences, RMIT University, GPO Box 2476V Melbourne, Victoria 3001, Australia

Running title: The forced radiation efficiency of panels

${ }^{a}$ Portions of this work were presented and published in "The radiation efficiency of finite size flat panels," Acoustics 2004, Transportation Noise and Vibration - The New Millennium, Proceedings of the Annual Conference of the Australian Acoustical Society, Gold Coast, November 3-5, 2004.

${ }^{\mathrm{b}}$ Electronic mail: john.davy@rmit.edu.au .

${ }^{c}$ Also employed at CSIRO Materials Science and Engineering, PO Box 56 Highett Victoria 3190, Australia,john.davy@csiro.au . 
The radiation efficiency of an infinite flat panel which is radiating a plane wave into a half space is equal to the inverse of the cosine of the angle between the direction of propagation of the plane wave and the normal to the panel. The fact that this radiation efficiency tends to infinity as the angle tends to $90^{\circ}$ causes problems with simple theories of sound insulation. Sato has calculated numerical values of radiation efficiency for a finite size rectangular panel in an infinite baffle whose motion is forced by sound incident at an angle to the normal from the other side. This paper presents a simple two dimensional analytic strip theory which agrees reasonably well with Sato's numerical calculations for a rectangular panel. This leads to the conclusion that it is mainly the length of the panel in the direction of radiation, rather than its width that is important in determining its radiation efficiency. A low frequency correction is added to the analytic strip theory. The theory is analytically integrated over all angles of incidence, with the appropriate weighting function, to obtain the diffuse sound field forced radiation efficiency of a panel.

PACS numbers: 43.55.Rg, 43.40.Rj, 43.20.Rz, 43.55.Ti 


\section{NOMENCLATURE}

a Half length of source

c Speed of sound in air

$f \quad$ Empirical limit of range of validity of equations (10) and (11)

$g \quad$ Cosine of angle of incidence

$g_{l} \quad$ Theoretical limit of range of validity of equations (10) and (11)

$h \quad$ Inverse of radiation efficiency at grazing angles

H Function

$H_{1} \quad$ Function

$\mathrm{H}_{2} \quad$ Function

$k \quad$ Wave number in air

$k_{b} \quad$ Wave number in panel

$L \quad$ Half shorter side length of rectangle

$m \quad$ Constant

$n \quad$ Empirical combining power

$N \quad$ Number of sound sources

$p \quad$ Sound pressure amplitude in air

$q \quad$ Inverse of real part of low frequency radiation efficiency

$r \quad$ Radius of sphere or hemisphere

$S \quad$ Surface area

$t \quad$ Time

U Perimeter

w Empirical correction factor for the limit of range of validity of equations (10) and (11) 
$W \quad$ Half longer side length of rectangle

$x \quad$ Variable of integration, or aspect ratio of rectangle

$y \quad$ Complement of angle of incidence

$Z_{w f} \quad$ Fluid wave impedance of panel in air

$\alpha \quad$ Interpolation slope

$\beta \quad$ Empirical offset correction for radiation efficiency at grazing incidence

$\delta \quad$ Half total phase change at observer

$\theta \quad$ Angle of radiation relative to normal

$\lambda \quad$ Wavelength in air

$\rho_{0} \quad$ Ambient density of air

$\sigma \quad$ Radiation efficiency

$\varphi \quad$ Angle of incidence relative to normal

$\varphi_{l} \quad$ Limiting angle of incidence relative to normal

$\psi \quad$ Half change of phase across source

$\omega \quad$ Angular frequency

$\omega_{c} \quad$ Angular critical frequency

\section{INTRODUCTION}

Below the critical frequency of a panel, the sound transmission through the panel is due mainly to the radiation of sound from the forced bending waves which are excited by sound incident on the other side. This is because the free bending waves generated by the reflection of the forced bending waves from the edges of the panel have wavelengths which are shorter than the wavelength of sound in air. This means that these free bending waves are very inefficient radiators of sound. At and above the critical frequency of the panel, the free bending waves in 
the panel have wavelengths which are the same as the forced bending waves excited by sound incident at a frequency dependent angle. Thus to predict the sound insulation of a panel it is necessary to know the radiation efficiency of forced bending waves propagating in the panel.

If a plane wave strikes a panel it forces a bending wave in the panel whose wavelength is greater than or equal to the wavelength of the incident wave in air. Because of this, the forced wave in the panel can radiate efficiently into air on its other side. The radiation efficiency of an infinite panel is equal to the inverse of the cosine of the angle of incidence and transmission (see section IV.7.a of Cremer and Heckl (1988)). This result obviously cannot be correct for a finite size panel because it goes to infinity at grazing incidence.

Gösele derived the radiation efficiency for an infinite strip in 1953 (Cremer and Heckl, 1988). Sato made numerical calculations in 1973 of the radiation efficiency of a forced wave on a square panel for case where the panel wavelength is longer than the wavelength of sound in air. Sato also numerically calculated the radiation efficiency averaged over all possible directions of sound incidence. Sato's results appear as Fig. 1.3.2, Table B.1 and Fig. B.2 in Rindel (1975).

Rindel (1975) uses Sato's numerical results for radiation efficiency in his theory of sound insulation as a function of angle of incidence. According to Novak (1992), Lindblad produced an approximate formula for the radiation efficiency at high frequencies in 1973 based on Gösele's results. Lindblad derived a simpler approximation in 1985 which can be integrated over all angles of incidence. He also extended the integrated formula to low frequencies.

Rindel (1993a) modified Lindblad's 1973 formula, with constants which are selected to provide good agreement with Sato's tabulated radiation efficiencies. Rindel's formula also extended Lindblad's 1973 formula to low frequencies. This formula of Rindel cannot be integrated by analytic means. 
Ljunggren (1991) repeated Sato's numerical calculations using a two dimensional model and obtains agreement "well within $0.5 \mathrm{~dB}$ ", both as a function of angle of incidence and averaged over all angles of incidence. Novak (1995) performed even more extensive three dimensional calculations than Sato.

The purpose of this paper is to derive an analytic approximation to Sato's numerical results using a simple two dimensional strip model. This analytic approximation has to be simple enough so that it can be integrated by analytic means over all angles of incidence for comparison with Sato's diffuse field results.

\section{DISCRETE AND LINE SOURCES}

In this section the radiation of sound from discrete sound sources on a line into a two dimensional plane is considered. This problem is generalised to obtain the radiation from a continuous line source in this section and the radiation from an infinite strip in the next section.

Fig. 1 shows two point sound sources which are separated by a distance $2 a$ which is shown as a solid line. The two sound sources emit pure tones with equal frequency and equal amplitude. An observer at a distance which is very large compared to the distance $2 a$ which separates the sound sources receives almost the same amplitude sound wave from each source. The lines from the two sound sources to the distant observer, which are shown with long dashes, are almost parallel.

The sound wave from source 1 travels an extra distance $2 a \sin \theta$, where $\theta$ is the angle between the normal, shown with short dashes, to the line joining the two sound sources and the parallel lines from the two sources to the distant observer. It is also assumed that the phase of source 2 leads the phase of source 1 by $2 \psi$. Thus at the distant observer, the phase of the sound from source 2 leads the phase of the sound from source 1 by $2 \delta=2 \psi+2 k a \sin \theta$ 
Now assume that there are $N$ sources of angular frequency $\omega$ in a line of length $2 a$. Each source has an amplitude proportional to $1 / N$, is a distance $2 a /(N-1)$ from the previous source and leads the phase of the previous source by $2 \psi /(N-1)$. At the distant observer, the phase of the sound from each source leads the phase of the sound from the previous source by

$$
2 \delta=\frac{2 \psi+2 k a \sin \theta}{N-1} .
$$

At time $t$, the sound pressure at the distant observer is proportional to

$$
\frac{1}{N} \sum_{n=1}^{N} \sin [\omega t+2(n-1) \delta]=\frac{\sin (N \delta)}{N \sin (\delta)} \sin [\omega t+(N-1) \delta] .
$$

The above summation is performed using formula 1.341 .1 on page 29 of Gradshteyn and Ryzhik (1965).

If $N$ is very large

$$
N \delta \approx(N-1) \delta=\psi+k a \sin \theta
$$

Thus

$$
\delta=\frac{\psi+k a \sin \theta}{(N-1)} \ll 1
$$

and $\sin \delta=\delta$.

Thus the sound pressure at the distant observer is proportional to

$$
\frac{\sin (N \delta)}{N \sin (\delta)} \sin [\omega t+(N-1) \delta]=\frac{\sin (\psi+k a \sin \theta)}{\psi+k a \sin \theta} \sin (\omega t+\psi+k a \sin \theta) .
$$

This large $N$ limit gives the result for a continuous line source of constant source strength over a length of $2 a$ and phase difference which varies linearly by a total amount of $2 \psi$ over the length $2 a$ of the continuous line source. The sound pressure amplitude $p$ at a distant observer is proportional to 


$$
p \propto \frac{\sin (\psi+k a \sin \theta)}{\psi+k a \sin \theta} .
$$

If the phase difference $\psi$ is due to a forced bending wave induced by a wave incident at an angle of $\varphi$

$$
\psi=-k_{b} a=-k a \sin \varphi
$$

In this case the sound pressure amplitude $p$ at a distant observer is proportional to

$$
p \propto \frac{\sin [k a(\sin \theta-\sin \varphi)]}{k a(\sin \theta-\sin \varphi)} .
$$

\section{INFINITE STRIPS}

Consider an infinite strip of width $2 a$ and ask how much power per unit length it radiates from one side when excited by a pure tone wave incident at an angle of $\varphi$ to the normal to the strip. The wave maxima planes are assumed to be parallel to the two parallel edges of the strip. This is the two dimensional problem considered in the previous section. Squaring the amplitude given by eq. (8) produces the power which can be summed by integrating over all angles of radiation $\theta$ from $-\pi / 2$ rad to $\pi / 2$ rad. Make the following approximation

$$
\sin \theta-\sin \varphi=2 \sin \left(\frac{\theta-\varphi}{2}\right) \cos \left(\frac{\theta+\varphi}{2}\right) \approx(\theta-\varphi) \cos \varphi \text { for }|\theta-\varphi| \ll 1
$$

If the approximation given by eq. (9) is inserted into the square of eq. (8), the resulting expression is only significantly different from zero when $\varphi$ is near $\theta$. Because $\theta$ is between $-\pi / 2$ and $\pi / 2$, it is possible to approximate by extending the limits of integration to $-\infty$ to $\infty$. These approximations will give the infinite panel result. Later in this paper, the range of validity of these approximations is examined and other approximations are obtained for use outside the range of validity of these approximations. A low frequency correction will also be introduced in 
the next section. With the above approximations the total radiated sound power per unit length of strip is proportional to

$$
\int_{-\infty}^{\infty} \frac{\sin ^{2}[k a(\theta-\varphi) \cos \varphi]}{[k a(\theta-\varphi) \cos \varphi]^{2}} d \theta=\int_{-\infty}^{\infty} \frac{\sin ^{2}(k a \theta \cos \varphi)}{(k a \theta \cos \varphi)^{2}} d \theta=\frac{\pi}{k a \cos \varphi}
$$

using integral 3.821.9 on page 446 of Gradshteyn and Ryzhik (1965).

This is the same $1 / \cos \varphi$ variability as in the case of the infinite panel since for the infinite panel, the transmitted angle $\theta$ is equal to the incident angle $\varphi$. Eq. (10) is only proportional to the radiation efficiency of the infinite strip. Since the radiation efficiency of an infinite strip must equal the $1 / \cos \varphi$ radiation efficiency of an infinite panel if $k a$ is large enough, eq. (10) must be multiplied by $k a / \pi$ to obtain the absolute value of the radiation efficiency. Thus the radiation efficiency of an infinite strip for an incident angle of $\varphi$ is

$$
\sigma(\varphi)=\frac{1}{\cos \varphi}=\frac{1}{g}
$$

where $g=\cos \varphi$. This result is obtained by Gösele, Lyon and Maidanik (1962), and Maidanik (1962).

The range of validity of eq. (11) is now investigated. The maximum value of the integrand on the left side of eq. (10) is 1 when $\theta=\varphi$. Thus replace this integrand in eq. (10) with a function which is equal to 1 when

$$
|\theta-\varphi| \leq \frac{\pi}{2 k a \cos \varphi}
$$

and is zero elsewhere. This function gives the same value for the integral. For this replacement function the change to the limits of integration is only valid if the nonzero part of the replacement function lies between $-\pi / 2$ to $\pi / 2$. This means that

$$
\frac{\pi}{2}-|\varphi| \geq \frac{\pi}{2 k a \cos \varphi}
$$


For $|\varphi|$ close to $\pi / 2$, the left hand side of equation (13) is approximately $\cos \varphi$. Thus eq. (13) becomes

$$
\cos \varphi \geq \frac{\pi}{2 k a \cos \varphi}
$$

or

$$
\cos \varphi \geq \sqrt{\frac{\pi}{2 k a}},
$$

or

$$
|\varphi| \leq \arccos \sqrt{\frac{\pi}{2 k a}} .
$$

Thus eq. (10) and (11) are only valid in the range given by eq. (16). At the two angles of incidence $\varphi_{l}$ given by the equal sign in eq. (16), the total radiated sound power per unit length of the strip is proportional to

$$
\frac{\pi}{k a \cos \varphi_{l}}=\frac{\pi}{k a} \sqrt{\frac{2 k a}{\pi}}=\sqrt{\frac{2 \pi}{k a}} .
$$

For later use, eq. (15) is rewritten as

$$
g=\cos \varphi \geq\left\{\begin{array}{ll}
g_{l}=\sqrt{\frac{\pi}{2 k a}} & \text { if } g_{l} \leq 1 \\
1 & \text { if } g_{l}>1
\end{array},\right.
$$

where $g_{l}$ is defined by the second equality in eq. (18).

In practice, an empirical correction factor $w$ is used in eq. (18) for the range of eq. (10) and (11).

$$
g=\cos \varphi \geq f= \begin{cases}w g_{l}=w \sqrt{\frac{\pi}{2 k a}} & \text { if } w g_{l} \leq 1 \\ 1 & \text { if } w g_{l}>1\end{cases}
$$


where $f$ is defined by the second equality in eq. (19). The empirical correction factor $w$ will be determined by comparison with Sato's numerical calculations for a square panel.

Since the maximum value of the integrand on the left side of eq. (10) is one, the maximum value of the integral before extending the limits is $\pi / 2-(-\pi / 2)=\pi$. Also $\cos \varphi$ is in the range from 0 to 1 for all values of $\varphi$ in the range from $-\pi / 2$ to $\pi / 2$. Thus we have

$$
\frac{\pi}{k a} \leq \frac{\pi}{k a \cos \varphi} \leq \pi .
$$

This means that the approximations can only be valid if $k a \geq 1$.

It is also possible to approximate the integral if $|\varphi|=\pi / 2$. Because of symmetry in the equations, only the case $\varphi=\pi / 2$ is considered. Now

$$
\sin (\theta)-\sin (\varphi)=\cos \left(\frac{\pi}{2}-\theta\right)-1 .
$$

If $\pi / 2-\theta$ is small, eq. (21) becomes

$$
1-\frac{1}{2}\left(\frac{\pi}{2}-\theta\right)^{2}-1=-\frac{1}{2}\left(\frac{\pi}{2}-\theta\right)^{2} .
$$

Put $y=\pi / 2-\theta$, then

$$
k a[\sin (\theta)-\sin (\varphi)]=-k a y^{2} / 2 .
$$

The integral in eq. (10) becomes

$$
\int_{0}^{\infty} \frac{\sin ^{2}\left(k a y^{2} / 2\right)}{\left(k a y^{2} / 2\right)^{2}} d y
$$

The $\theta=\pi / 2$ limit has become $y=0$. The $\theta=-\pi / 2$ limit has become $y=\pi$ and been extended to $y$ $=\infty$. This extension will be compensated by the introduction of an empirical offset correction $\beta$ in eq. (27) and the introduction of a low frequency correction in the next section. Using integral number 3.852 .3 on page 464 of Gradshteyn and Ryzhik (1965), eq. (24) becomes 


$$
\left(\frac{2}{k a}\right)^{2} \frac{2 \sqrt{\pi}}{3}\left(\frac{k a}{2}\right)^{3 / 2}=\frac{2}{3} \sqrt{\frac{2 \pi}{k a}} .
$$

It should be noted that this result is $2 / 3$ of the value derived in eq. (17) for when

$$
\cos \varphi_{l}=\sqrt{\frac{\pi}{2 k a}}
$$

Like eq. (10), eq. (25) must be multiplied by $k a / \pi$ to obtain the absolute value of the radiation efficiency. The radiation efficiency of an infinite strip for a grazing incident angle $\varphi$ for which $|\varphi|$ $=\pi / 2 \mathrm{rad}$ is

$$
\sigma\left(\frac{\pi}{2}\right)=\frac{1}{h}=\frac{2}{3} \frac{1}{g_{l}}-\beta=\frac{2}{3} \sqrt{\frac{2 k a}{\pi}}-\beta
$$

where

$$
h=\frac{1}{\frac{2}{3 g_{l}}-\beta}=\frac{1}{\frac{2}{3} \sqrt{\frac{2 k a}{\pi}}-\beta},
$$

and $\beta$ is an empirical offset correction which is determined by comparison with Sato's numerical calculations for a square panel. It is thought that $\beta$ is needed because the theory is developed for an infinite strip but is applied to the case of a square panel. $\beta$ accounts for the fact that the radiation efficiency of an infinite strip is slightly greater than that of a square panel at grazing angles of excitation.

This result with $\beta=0$ is derived by Gösele. Lyon and Maidanik (1962) also derive this result with $\beta=0$, but a factor of 2 appears to be missing from their equation. Cremer and Heckl (1988) insert the factor of 2 when quoting Lyon and Maidanik's result. Maidanik (1962) derives this result with $\beta=0$ but then appears to include an extra factor of $2 \times 1.06=2.12$ in his final result for the radiation efficiency at grazing angles of incidence. The source of the 1.06 is explained by eq. (31) and (32) of this paper. This over estimate of Maidanik (1962) in his final 
result for the radiation efficiency at grazing angles of incidence is confirmed by Novak's (1995) three dimensional numerical calculations on a square panels of side length $2 a$ for $k a=16$ and $k a$ $=64$. For a square panel with sides of length $2 a$, Maidanik's (1962) final result for the radiation efficiency at grazing angles of incidence is

$$
\langle\sigma\rangle=\sqrt{\frac{2 a}{\lambda}}+\sqrt{\frac{2 a}{\lambda}}=2 \sqrt{\frac{2 a}{\lambda}} .
$$

For a square panel with sides of length $2 a$, Cremer and Heckl (1988) quote Maidanik's (1962) final result for the radiation efficiency at grazing angles of incidence as

$$
\langle\sigma\rangle=0.45 \sqrt{\frac{U}{\lambda}}=0.45 \sqrt{\frac{8 a}{\lambda}}=0.9 \sqrt{\frac{2 a}{\lambda}},
$$

where $U$ is the perimeter of the panel. The ratio of eq. (29) to eq. (30) of $2 / 0.9=2.22$ is close to the extra factor of 2.12 which appears to be included in eq. (29). Leppington et al. (1982) also show that Maidanik's (1962) final result for the radiation efficiency at grazing angles of incidence for rectangular panels with aspect ratios between 0.3 and 1 overestimates "by a factor of about 2." It should be noted that Maidanik (1962) only claims that his "values should be accurate at least to an order of magnitude."

$$
\text { If } \beta=0 \text {, }
$$

$$
h=\frac{3}{2} \sqrt{\frac{\pi}{2 k a}}=\frac{3}{2 \sqrt{2}} \sqrt{\frac{\lambda}{2 a}}=1.06 \sqrt{\frac{\lambda}{2 a}} .
$$

Because 1.06 is approximately equal to one, Maidanik (1962), Lindblad and Novak (1992) use the following approximation.

$$
h=\sqrt{\frac{\lambda}{2 a}} \text { and } \sigma\left(\frac{\pi}{2}\right)=\frac{1}{h}=\sqrt{\frac{2 a}{\lambda}} .
$$


The range of eq. (11) is extended beyond that given by eq. (19) by combining the infinite panel result of $1 / \cos \varphi$ (see eq. (11)) with the infinite strip result at a grazing angle of incidence of eq. (27). Linear interpolation in $g=\cos \varphi$ in the denominator of eq. (11) from $f$ when $g=\cos \varphi$ $=f$ to $h$ when $g=\cos \varphi=0$ is used. This gives

$$
\sigma(g)=\left\{\begin{array}{ll}
\frac{1}{g} & \text { if } 1 \geq|g| \geq f \\
\frac{1}{h-\alpha g} & \text { if } f>|g| \geq 0
\end{array},\right.
$$

where the interpolation slope $\alpha$ is chosen so that the two parts of eq. (33) are equal when $g=\cos$ $\varphi=f$. This gives

$$
\alpha=\frac{h}{f}-1
$$

\section{FINITE SIZE PANELS}

The approximation of extending the limits of integration from $-\pi / 2$ and $\pi / 2$ to $-\infty$ and $\infty$ in eq. (10) and from $\pi$ to $\infty$ in eq. (24) means that eq. (33) will over estimate the radiation efficiency at low frequencies where $k a$ is small. To extend the results to values of $k a$ less than one, assume a finite size panel of area $S$. Since only the power that is radiated is of interest, only the real part of the fluid wave impedance $Z_{w f}$ is considered. For a symmetrically pulsating sphere of radius $r$, the real part of the normalised fluid wave impedance for $k r<<1$ is $k^{2} r^{2}$. By symmetry this result also applies to a pulsating hemisphere whose centre is on an infinite rigid plane. For sources whose size is small compared to the wavelength of sound, it is expected that the real parts of their normalised fluid wave impedances will depend only on their areas. Thus the result for the pulsating hemisphere also applies to a panel set in an infinite rigid plane baffle providing the area of the panel is equal to the surface area of the hemisphere. Thus $2 \pi r^{2}=S$ where $S$ is the area of 
the panel. For a square panel of side length $2 a$ the area of the panel is $S=4 a^{2}$. The radiation efficiency of the panel is

$$
\sigma=\frac{\operatorname{Re}\left(Z_{w f}\right)}{\rho_{0} c}=k^{2} r^{2}=\frac{k^{2} S}{2 \pi}=\frac{1}{q}=\frac{2}{\pi} k^{2} a^{2},
$$

where the inverse of the radiation efficiency of the panel is

$$
q=\frac{2 \pi}{k^{2} S}=\frac{\pi}{2 k^{2} a^{2}} .
$$

Eq. (35) and (36) depend on the assumption that all areas of the square panel are vibrating in phase with uniform amplitude. For a panel that is excited by incident airborne sound and whose dimensions are small compared to the wavelength of sound in air, this is a reasonable assumption. For non-square panels, the characteristic length to use in eq. (35) and (36) is $2 a=\sqrt{S}$ where $S$ is the area of the panel.

Eq. (33) and (35) are combined by inverting, raising to the power of $n$, adding, taking the $n$th root and inverting. Lindblad, Novak (1992) and Rindel (1993a) obtain their versions of eq. (33) by using this method to combine the two different incident angle ranges. Rindel (1993a) also used it to combine his version of eq. (33) with eq. (35). This combining method gives the radiation efficiency as

$$
\sigma(g)=\left\{\begin{array}{ll}
\frac{1}{\sqrt[n]{g^{n}+q^{n}}} & \text { if } 1 \geq|g| \geq f \\
\frac{1}{\sqrt[n]{[h-\alpha g]^{n}+q^{n}}} & \text { if } f>|g| \geq 0
\end{array} .\right.
$$

The empirical combining power $n$ will be determined by comparison with Sato's numerical calculations for a square panel.

\section{AVERAGE OVER AZIMUTHAL ANGLE}


For a specific azimuthal incidence direction, $2 a$ should be set equal to a characteristic length of the panel in the azimuthal incidence direction. For averages over all azimuthal incidence directions, Ljunggren (1991) suggests that $2 a$ should be set equal to the mean free path $\frac{\pi S}{U}$ where $S$ is the area and $U$ is the perimeter of the panel. Ljunggren (1991) also shows that the root mean square length across a rectangle is equal to $\sqrt{\frac{2 S}{\pi}}$ and points out that this "is in most cases, in practice, fairly close to the mean free path". Novak (1995) confirms the usefulness of Ljunggren's (1991) suggestion by showing that the diffuse field forced radiation efficiencies of a square panel and a rectangular panel are almost identical for the same value of $\frac{k \pi S}{U}$.

The problem with $\frac{\pi S}{U}$ and $\sqrt{\frac{2 S}{\pi}}$ is that they are equal to $\frac{\pi}{4} 2 a=0.785 \times 2 a$ and $\sqrt{\frac{2}{\pi}} 2 a=0.798 \times 2 a$ respectively, rather than $2 a$, for a square panel with side length $2 a$. All that Novak's (1995) result actually shows is that the characteristic length should be proportional to $\frac{S}{U}$. Rindel (1993a, 1993b) follows Thomasson's (1982) proposal of a characteristic length of $\frac{4 S}{U}$. This is consistent with Novak's (1995) result and is equal to $2 a$ for a square panel with side length $2 a$. It is also equal to the diameter for a circular panel.

As shown in eq. (30), Cremer and Heckl (1988) use a characteristic length of $\frac{U}{4}$ when quoting Maidanik's (1962) final result for the radiation efficiency at grazing angles of incidence. This is equal to $2 a$ for a square panel with side length $2 a$. Sewell (1970) uses a characteristic length of $\sqrt{S}$. This is also equal to $2 a$ for a square panel with side length $2 a$ and is the 
characteristic length which is used in eq. (35) and (36). One of Maidanik's (1962) equations can also be interpreted as using a characteristic length of $\sqrt{S}$ at grazing incidence. However, Sewell (1970) also uses a correction offset for non-square rectangular panels. This shows that $\sqrt{S}$ is not completely satisfactory as a characteristic length for non-square panels. Thus, this paper recommends the use of a characteristic length of $2 a=\frac{4 S}{U}$ for non-square panels, except in eq. (35) and (36), where the characteristic length $2 a=\sqrt{S}$ is used.

Leppington et al. (1982) show that the radiation efficiency, at grazing incidence averaged over all azimuthal angles of incidence, of a rectangular panel with a shorter side length of $2 L$ and a longer side length of $2 W$ is

$$
\sigma=H(x) \sqrt{2 k L} \text { where } x=\frac{2 L}{2 W} \text {. }
$$

Leppington et al. (1982) give a formula for $H(x)$ which involves a fairly complicated integral and a graph of $H(x)$ for $x$ between 0 and 1 . They say that the approximation $H_{1}(x)=0.5-0.15 x$ differs from $H(x)$ by less than $4 \%$. For the same rectangular panel and a characteristic length of $2 a=\frac{4 S}{U}=\frac{4 L}{1+x}$, eq. (27), with $\beta=0$, can be written as

$$
\sigma\left(\frac{\pi}{2}\right)=H_{2}(x) \sqrt{2 k L} \text { where } H_{2}(x)=\frac{2}{3} \sqrt{\frac{2}{\pi}} \frac{1}{\sqrt{1+x}} \text { and } x=\frac{2 L}{2 W}
$$

$H_{2}(x)$ differs from $H(x)$ by less than about $5 \%$ and from $H_{1}(x)$ by less than about $6 \%$. Thus the work of Leppington et al. (1982) also justifies the use of a characteristic length of $2 a=\frac{4 S}{U}$.

\section{AVERAGE OVER ANGLE OF INCIDENCE}

The radiation efficiency averaged over all angles of incidence $\varphi$ and all azimuthal angles is 


$$
\langle\sigma\rangle=\int_{0}^{\pi / 2} \sigma(\varphi) \sin \varphi d \varphi
$$

The $\sin \varphi$ occurs in the integral because there is more solid angle for sound to be incident from the closer $\varphi$ is to $\pi / 2$. To evaluate this integral, assume the constant value of the characteristic $2 a=\frac{4 S}{U}$ given in the previous section.

Differentiating $g=\cos \varphi$ gives $d g=-\sin \varphi d \varphi$. Eq. (40) becomes

$$
\langle\sigma\rangle=\int_{f}^{1} \frac{d g}{\sqrt[n]{g^{n}+q^{n}}}+\int_{0}^{f} \frac{d g}{\sqrt[n]{(h-\alpha g)^{n}+q^{n}}}
$$

If $\alpha \neq 0$, then put $x=h-\alpha g$. Differentiating gives $d x=-\alpha d g$ and eq. (41) becomes

$$
\langle\sigma\rangle=\int_{f}^{1} \frac{d g}{\sqrt[n]{g^{n}+q^{n}}}+\frac{1}{\alpha} \int_{f}^{h} \frac{d x}{\sqrt[n]{x^{n}+q^{n}}} .
$$

If $n=2$, integral number 2.271 .4 on page 86 of Gradshteyn and Ryzhik (1965) gives

$$
\langle\sigma\rangle=\ln \left(\frac{1+\sqrt{1+q^{2}}}{f+\sqrt{f^{2}+q^{2}}}\right)+\frac{1}{\alpha} \ln \left(\frac{h+\sqrt{h^{2}+q^{2}}}{f+\sqrt{f^{2}+q^{2}}}\right) .
$$

If $\mathrm{n}=1$, integral number 2.111.1 on page 58 of Gradshteyn and Ryzhik (1965) gives

$$
\langle\sigma\rangle=\ln \left(\frac{1+q}{f+q}\right)+\frac{1}{\alpha} \ln \left(\frac{h+q}{f+q}\right) .
$$

Another possible way of combining the infinite panel result with the infinite strip result for grazing incidence, which was introduced by Lindblad in 1985, is obtained by setting $\alpha=0$ and

$$
f=\left\{\begin{array}{l}
h \text { if } h \leq 1 \\
1 \text { if } h>1
\end{array} .\right.
$$

This paper will describe this method as Lindblad's simpler method.

If $\alpha=0$, eq. (43) and (44) must be replaced by the following two equations respectively. For $n=2$ 


$$
\langle\sigma\rangle=\ln \left(\frac{1+\sqrt{1+q^{2}}}{f+\sqrt{f^{2}+q^{2}}}\right)+\frac{f}{\sqrt{h^{2}+q^{2}}} .
$$

For $n=1$

$$
\langle\sigma\rangle=\ln \left(\frac{1+q}{f+q}\right)+\frac{f}{h+q} .
$$

Lindblad also combines the infinite panel result with the infinite strip result at grazing incidence using the same combining method as used to obtain eq. (37) in this paper. That is, he inverts, raises to the power $n$, sums, takes the $n$th root and inverts again. Lindblad uses $n=4$. This paper will describe this method as Lindblad's more complicated method. Novak (1992) uses this method with $n=10$. The disadvantage of using this method with $n=4$ or 10 is that it is not easy to analytically evaluate the integral of eq. (40). Lindblad only applies the low frequency correction to the integrated version of his simpler approximation. Lindblad and Novak (1992) do not combine their results with the low frequency result of eq. (35). In this paper their results are combined with the low frequency result using $n=2$.

Rindel's approximation (Rindel, 1993a) is the only other formula to produce a peak at other than grazing incidence or at normal incidence. It is also the only other formula to include the low frequency correction $q$. It combines the low angle of incidence result with both the grazing incidence result and the low frequency result using the $n=4$ method, but modifies the low angle of incidence result and the grazing incidence result so that the maximum occurs at $g=$ $1.1 g_{l}$. This compares with the method developed in this paper which has the maximum at $g=1.3$ $g_{l}$. The observation that Rindel's maximum occurs at a value of $g$ different to $g_{l}$ is one of the reasons for the introduction of the empirical correction factor $w$ in this paper. Unfortunately Rindel's formula is too complicated to be easily analytically integrated. This is why Rindel (1993b) also develops a separate approximation to the diffuse field radiation efficiency. 
For comparison with some other results, ignore the low frequency result by setting $q=0$, set $w=1$ and $\beta=0$, and assume that $f \leq 1$. These assumptions give $h / f=3 / 2$ and eq. (43) and (44) both reduce to

$$
\langle\sigma\rangle=\frac{1}{2}(\ln 2 k a+0.477)
$$

Rindel (1993b) gives the following diffuse field radiation efficiency approximation which he says is useful for $k a>0.5$.

$$
\langle\sigma\rangle=\frac{1}{2}(\ln 2 k a+0.2)
$$

Sewell's (1970) work can be interpreted as producing a similar formula with a low frequency correction. This is because the diffuse field sound transmission coefficient for a single isotropic panel is obtained by multiplying the panel's normal incidence transmission coefficient by twice eq. (40).

$$
\langle\sigma\rangle=\frac{1}{2}\left(\ln 2 k a+0.160+\frac{1}{16 \pi k^{2} a^{2}}\right) .
$$

Setting $k a=1$ in the third term in the brackets in equation (63) gives

$$
\langle\sigma\rangle=\frac{1}{2}(\ln 2 k a+0.180)
$$

Sewell (1970) also gives a correction for non-square rectangular panels which reduces the diffuse field radiation efficiency.

Adopting Lindblad's assumptions of $q=0, \alpha=0, f$ given by eq. (45), $h$ given by eq. (32), and $f \leq 1$, gives $f=h$ and eq. (46) and (47) both reduce to Lindblad's equation for the diffuse field radiation efficiency.

$$
\langle\sigma\rangle=\ln \left(\sqrt{\frac{k a}{\pi}}\right)+1=\frac{1}{2}(\ln 2 k a+0.162) .
$$


If Sewell's low frequency correction term is ignored, his eq. (50) is almost the same as Lindblad's eq. (52) and very similar to Rindel's eq. (49). Comparison of these three equations with eq. (48) suggests that eq. (48) slightly over estimates the diffuse field radiation efficiency. This is one of the reasons for applying the offset correction term $\beta$ to the grazing incidence radiation efficiency.

Eq. (37) of this paper can also be used to predict the sound radiation of free bending waves in a panel if the angular frequency $\omega$ is greater than or equal to the angular critical frequency of the panel $\omega_{c}$. Because the free bending wavelength of the panel is greater than or equal to the wavelength of sound in air $\lambda$ and correspondingly the free bending wave number of the panel $k_{b}$ is less than or equal to the wave number of sound in air $k$, $\sin \theta$ can be calculated from $k_{b}=k \sin \theta$. This enables $g=\cos \theta$ to be calculated.

$$
g=\cos \theta=\sqrt{1-\sin ^{2} \theta}=\sqrt{1-\left(\frac{k_{b}}{k}\right)^{2}}=\sqrt{1-\frac{\omega_{c}}{\omega}} .
$$

Note that equation (2.39c) in Maidanik (1962) mistakenly interchanges the critical frequency and the frequency. This mistake is not corrected in the errata.

\section{COMPARISON WITH CALCULATION AND EXPERIMENT}

Davy (2004) used $n=1, w=1$ and $\beta=0$ and in the associated conference presentation gave results for the case $n=2, w=1$ and $\beta=0$. This paper uses $n=2, w=1.3$ and $\beta=0.124$ because these values produce the best agreement with Sato's numerically calculated results. Figure 2 shows the calculated values of the radiation efficiency in $\mathrm{dB}$ as a function of the angle of incidence from $0^{\circ}$ to $90^{\circ}$ for values of $k a$ ranging from 0.5 to $\infty$.

Sato produced a table of numerically calculated values of the forced radiation efficiency in $\mathrm{dB}$ to one decimal place for a square panel with side length $2 a$, at $15^{\circ}$ increments of the angle 
of incidence from $0^{\circ}$ to $90^{\circ}$ to the normal to the panel for 15 values of $k a$ from 0.5 to 64 inclusive. Table I shows the difference in $\mathrm{dB}$ between eq. (37) and Sato's numerical results. The biggest errors occur because of ripple in the change from the high frequency region to the low frequency region around $k a=2$. This is why most other authors do not extended their approximations to low frequencies. Table II shows the effect on the differences at an angle of incidence of $90^{\circ}$, if $\beta$ is changed from 0.124 to 0 .

Sato also graphed numerically calculated values of the forced radiation efficiency in $\mathrm{dB}$ for a square panel with side length $2 a$, at $5^{\circ}$ increments of the angle of incidence from $0^{\circ}$ to $90^{\circ}$ to the normal to the panel for six values of $k a$ from 2 to 64 inclusive. The extra values on this graph are read from Sato's graph and added to his tabulated values. This enlarged set of values is used to compare the results of eq. (37) (D), Rindel's (1993a) approximation (R1), Lindblad's more complicated approximation (L1), Lindblad's simpler approximation (L2), and Novak's (1992) approximation $(\mathrm{N})$ to Sato's numerically calculated results. The mean, the standard deviation, the maximum, and the minimum differences from Sato's numerically calculated results are shown in Table III. Eq. (37) performs slightly better than the other approximation formulae.

Figure 3 shows the calculated values of the diffuse field forced radiation efficiency in $\mathrm{dB}$ as a function $k a$ from 0.5 to 64 . Sato also tabulates numerically calculated values of the diffuse field forced radiation efficiency in $\mathrm{dB}$ to two decimal places, for a square panel with side length $2 a$, for fifteen values of $k a$ from 0.5 to 64 inclusive. Table IV shows the differences in $\mathrm{dB}$ between eq. (43) (D), Lindblad's diffuse field result from the integrated version of his simplified approximation (L2), Rindel's (1993b) diffuse field radiation efficiency approximation (R2), and Sewell's (1970) formula (S). The mean, the standard deviation, the maximum, and the minimum 
differences from Sato's numerically calculated results are also shown in Table IV. Rindel (1993b) says that his approximation is useful for $k a>0.5$. Sewell (1970) also gives a correction term for non-square rectangular panels. The integral of Lindblad's simpler approximation formula performs slightly better than the other formulae. If Lindblad's simpler approximation formula is integrated without the low frequency correction to obtain eq. (52) and the low frequency correction of eq. (35) is combined with it using the $n=2$ method, the result agrees with the L2 results in Table III to better than three decimal places.

Table IV shows that eq. (43) (D) is in good agreement with Sewell's (1970) formula. Sewell (1970) shows that his formula is in reasonable agreement with the sound insulation measurements of Utley (1986) in the Liverpool University facility on a limp lead panel, of surface density $17.6 \mathrm{~kg} / \mathrm{m}^{2}$, which measured $2.13 \mathrm{~m}$ by $1.67 \mathrm{~m}$. Thus eq. (43) is in reasonable agreement with Utley's (1986) measurements.

Another experimental verification of the theory of this paper is the fact that the author and his students (Pavasovic (2006), Davy and Pavasovic (2006), Davy and Kannanaikkel John (2006), Fisher (2006), Davy (2007), Davy and Kannanaikkel John (2007), and Davy (2008))

have been able to use the theory of this paper with $n=1, w=1$ and $\beta=0$ to successfully predict the experimental directivity of the forced sound radiation from panels and openings.

\section{CONCLUSIONS}

The two dimensional strip model analytic approximation derived in this paper gives reasonable agreement with three dimensional numerical calculations. This agrees with Ljunggren (1991) whose two dimensional numerical calculations agree within $\pm 0.5 \mathrm{~dB}$ of the three dimensional calculations of Sato and Novak (1995). It also agrees with the experimental measurements of Roberts (1983) which show that the directivity of a rectangle depends strongly 
on its length in the direction of measurement but only weakly on its width at right angles to the direction of measurement. Thus this paper concludes that the radiation efficiency of a forced wave on a panel is mainly determined by the ratio of the panel's length, in the direction of propagation of the forced wave, to the wavelength of the sound in air and the angle of incidence of the forcing wave.

It should be pointed out that the forced radiation of sound from an opening can be treated as the forced radiation of sound from a panel because, for the same source size and angle of incidence of the forcing wave, the radiation depends only on the velocity distribution across the opening or panel. Consequently the results from this paper may be applied to predicting the radiation of sound from openings as well.

\section{REFERENCES}

Cremer, L., and Heckl, M. (1988). Structure-Borne Sound : Structural Vibrations and sound radiation at audio frequencies, translated and revised by E. E. Ungar (Springer-Verlag Company, New York, 2nd edition).

Davy, J. L. (2004). "The radiation efficiency of finite size flat panels," Acoustics 2004, Transportation Noise and Vibration - The New Millennium, Proceedings of the Annual Conference of the Australian Acoustical Society, Gold Coast, Australia, 3-5 November, edited by M. J. Mee, R. J. Hooker, and I. D. M. Hillock, (Australian Acoustical Society, Castlemaine, Victoria, Australia), pp 555-560.

Davy, J. L. (2007). "A model for predicting diffraction on a finite flat surface as a function of angle of incidence and surface size," Global Approaches to Noise Control, Proceedings of the Inter-Noise 2007, the 36th International Congress and Exhibition on Noise Control Engineering, Istanbul, Turkey 28-31 August, (Turkish Acoustical Society, Istanbul, Turkey), Paper No. 21. 
Davy, J. L. (2008). "The directivity of the forced radiation of sound from panels and openings including the shadow zone", Proceedings of Acoustics'08, Paris, 29 June-4 July, pp 3833-3838, Paper No. 11. J. Acoust. Soc. of Am. 123, 3499(A), Acta Acustica united with Acustica, 94, S531(A).

Davy, J. L., and Kannanaikkel John, T. (2006). "The angular distribution of sound incident on a panel or opening (A),” J. Acoust. Soc. of Am. 120, 3187.

Davy, J. L., and Kannanaikkel John, T. (2007). "The directivity of the forced radiation of sound from panels and openings," Proceedings of the Fourteenth International Congress on Sound and Vibration (ICSV14), Cairns, Australia, 9-12 July, edited B. Randall, Paper No. 7.

Davy, J. L., and Pavasovic, V. (2006). "The directivity of sound radiated from a panel or opening excited by sound incident from the other side," Proceedings of Acoustics 2006, Noise of Progress, First Australasian Acoustical Societies' Conference, Christchurch, New Zealand, 2022 November, edited by T. McMinn, pp. 133-140.

Fisher, K. R. (2006). "Directivity of the sound transmission through finite sized apertures and panels," Honors dissertation, RMIT University, Melbourne, Australia), available at Arup Acoustics, Melbourne, Australia or the author of this paper.

Gradshteyn, I. S., and Ryzhik, I. M. (1965). Table of integrals, series, and products, Fourth Edition, prepared by Yu. V. Geronimus, and M. Yu. Tseytlin, , translated from Russian by Scripta Technica Inc., edited by A. Jeffrey (Academic Press, New York, $4^{\text {th }}$ edition).

Leppington, F. G., Broadbent, E. G., and Heron, K. H. (1982). “The acoustic radiation efficiency of rectangular panels," Proc. R. Soc. Lond. A 382, 245-271.

Ljunggren, S. (1991). "Airborne sound insulation of thin walls," J. Acoust. Soc. Am. 89, 23242337. 
Lyon, R. H., and Maidanik, G. (1962). "Power flow between linearly coupled oscillators," J. Acoust. Soc. Am. 34, 623-639.

Maidanik, G. (1962). "Response of ribbed panels to reverberant acoustic fields," J. Acoust. Soc. Am. 34, 809-826; Erratum: J. Acoust. Soc. Am. 57, 1552.

Novak, R. A. (1992). "Sound insulation of lightweight double walls," Applied Acoustics 37, 281-303.

Novak, R. A. (1995). “Radiation from partially excited plates,” Acta Acustica 3, 561-567.

Pavasovic, V. (2006). "The radiation of sound from surfaces at grazing angles of incidence" MS dissertation, RMIT University, Melbourne, Australia, available for download from the Australian Digital Theses Program http://adt.lib.rmit.edu.au/adt/public/adt-VIT20060911.115939 (Last viewed May 2009).

Rindel, J. H. (1975). "Transmission of traffic noise through windows - Influence of incident angle on sound insulation in theory and experiment," Report No. 9, The Acoustics Laboratory, Technical University of Denmark, Lyngby, Denmark.

Rindel, J. H. (1993a). "Modelling the angle-dependent pressure reflection factor," J. Applied Acoustics 38, 223-234.

Rindel, J. H. (1993b). "Sound transmission through single layer walls," in Noise-93, Proceedings of the International Conference on Noise and Vibration Control, St Petersburg, Russia, edited by M. J. Crocker, and N, I. Ivanov, pp. 63-68.

Roberts, J. (1983). “The prediction of directional sound fields," Transactions of the Institution of Engineers, Australia, Mechanical Engineering, ME8, 16-22.

Sewell, E. C. (1970). "Transmission of reverberant sound through a single-leaf partition surrounded by an infinite rigid baffle," J. Sound and Vib. 12, 21-32. 
Thomasson, S.-I. (1982). "Theory and experiments on the sound absorption as function of the area”, Report TRITA-TAK 8201, (Royal Institute of Technology, Stockholm, Sweden).

Utley, W. A. (1968). "Single leaf transmission loss at low frequencies," J. Sound and Vib. 8, 256-261. 
TABLE I. The differences (dB) between the radiation efficiency given by eq. (37) and Sato's numerically calculated radiation efficiency.

\begin{tabular}{|l|l|l|l|l|l|l|l|}
\hline $\mathrm{ka}$ & $0^{\circ}$ & $15^{\circ}$ & $30^{\circ}$ & $45^{\circ}$ & $60^{\circ}$ & $75^{\circ}$ & $90^{\circ}$ \\
\hline 0.5 & 0.3 & 0.3 & 0.2 & 0.2 & 0.2 & 0.1 & -0.1 \\
\hline 0.75 & 0.3 & 0.3 & 0.3 & 0.3 & 0.3 & 0.0 & -0.4 \\
\hline 1 & 0.3 & 0.3 & 0.3 & 0.3 & 0.2 & 0.0 & -0.4 \\
\hline 1.5 & -0.3 & -0.2 & -0.1 & 0.1 & 0.1 & 0.0 & -0.4 \\
\hline 2 & -0.7 & -0.6 & -0.4 & -0.1 & 0.0 & 0.1 & -0.1 \\
\hline 3 & 0.0 & -0.1 & -0.4 & -0.5 & -0.2 & 0.0 & -0.1 \\
\hline 4 & 0.3 & 0.1 & -0.1 & -0.3 & -0.2 & 0.0 & 0.0 \\
\hline 6 & 0.0 & -0.1 & 0.0 & -0.1 & -0.2 & 0.0 & 0.0 \\
\hline 8 & -0.1 & 0.0 & 0.1 & -0.1 & -0.1 & 0.0 & 0.0 \\
\hline 12 & 0.0 & 0.0 & 0.0 & 0.1 & -0.1 & -0.1 & 0.1 \\
\hline 16 & 0.0 & 0.0 & 0.0 & 0.0 & -0.2 & -0.1 & 0.1 \\
\hline 24 & 0.0 & 0.0 & 0.0 & 0.0 & 0.1 & -0.1 & 0.0 \\
\hline 32 & 0.0 & 0.0 & 0.0 & 0.0 & 0.0 & -0.1 & 0.0 \\
\hline 48 & 0.0 & 0.0 & 0.0 & 0.0 & 0.0 & -0.1 & 0.0 \\
\hline 64 & 0.0 & 0.0 & 0.0 & 0.0 & 0.0 & -0.2 & 0.0 \\
\hline
\end{tabular}


TABLE II. The differences (dB) between the radiation efficiency given by eq (37) and Sato's numerically calculated radiation efficiency for an angle of incidence of $90^{\circ}$ when $\beta=0.124$ and $\beta=0$.

\begin{tabular}{|l|l|l|}
\hline ka & $\beta=0.124$ & $\beta=0$ \\
\hline 0.5 & -0.1 & 0.3 \\
\hline 0.75 & -0.4 & 0.2 \\
\hline 1 & -0.4 & 0.3 \\
\hline 1.5 & -0.4 & 0.4 \\
\hline 2 & -0.1 & 0.6 \\
\hline 3 & -0.1 & 0.5 \\
\hline 4 & 0.0 & 0.5 \\
\hline 6 & 0.0 & 0.4 \\
\hline 8 & 0.0 & 0.4 \\
\hline 12 & 0.1 & 0.4 \\
\hline 16 & 0.1 & 0.3 \\
\hline 24 & 0.0 & 0.3 \\
\hline 32 & 0.0 & 0.2 \\
\hline 48 & 0.0 & 0.2 \\
\hline 64 & 0.0 & 0.1 \\
\hline
\end{tabular}


TABLE III. The mean, the standard deviation, the maximum, and the minimum differences (dB) of various approximations from Sato's numerically calculated radiation efficiency results.

\begin{tabular}{|l|l|l|l|l|l|}
\hline & $\mathrm{D}$ & $\mathrm{R} 1$ & $\mathrm{~L} 1$ & $\mathrm{~L} 2$ & $\mathrm{~N}$ \\
\hline mean & 0.0 & 0.0 & -0.2 & 0.0 & 0.0 \\
\hline stdev & 0.2 & 0.4 & 0.5 & 0.4 & 0.5 \\
\hline maximum & 0.3 & 0.9 & 0.8 & 0.8 & 0.8 \\
\hline minimum & -0.7 & -1.5 & -1.9 & -1.5 & -1.6 \\
\hline
\end{tabular}


TABLE IV. The differences $(\mathrm{dB})$ between various diffuse field radiation efficiency approximations and Sato's numerically calculated diffuse field radiation efficiency.

\begin{tabular}{|c|c|c|c|c|}
\hline$k a$ & D & L2 & R2 & $\mathrm{S}$ \\
\hline 0.5 & 0.18 & 0.20 & -1.50 & -0.72 \\
\hline 0.75 & 0.18 & 0.14 & 0.34 & 0.31 \\
\hline 1 & 0.15 & 0.05 & 0.29 & 0.19 \\
\hline 1.5 & 0.03 & -0.05 & 0.13 & 0.03 \\
\hline 2 & -0.11 & -0.03 & 0.14 & 0.05 \\
\hline 3 & -0.26 & -0.02 & 0.12 & 0.04 \\
\hline 4 & -0.11 & 0.06 & 0.16 & 0.08 \\
\hline 6 & -0.06 & 0.05 & 0.12 & 0.05 \\
\hline 8 & -0.03 & 0.05 & 0.11 & 0.05 \\
\hline 12 & 0.00 & 0.05 & 0.10 & 0.04 \\
\hline 16 & -0.02 & 0.02 & 0.06 & 0.01 \\
\hline 24 & -0.01 & 0.01 & 0.05 & 0.00 \\
\hline 32 & -0.03 & -0.01 & 0.02 & -0.02 \\
\hline 48 & -0.04 & -0.03 & 0.00 & -0.04 \\
\hline 64 & -0.05 & -0.05 & -0.02 & -0.05 \\
\hline mean & -0.01 & 0.03 & 0.01 & 0.00 \\
\hline stdev & 0.12 & 0.07 & 0.43 & 0.22 \\
\hline maximum & 0.18 & 0.20 & 0.34 & 0.31 \\
\hline minimum & -0.26 & -0.05 & -1.50 & -0.72 \\
\hline
\end{tabular}




\section{FIGURE CAPTIONS}

Figure 1. Two discrete sound sources.

Figure 2. The theoretical forced radiation efficiency of a square panel, with side length of $2 a$, as a function of the angle of incidence for a range of values of $k a$. These theoretical results have been calculated using eq. (19), (28), (34), (36), and (37).

Figure 3. The theoretical diffuse field forced radiation efficiency of a square panel, with side length of $2 a$, as a function of $k a$. These theoretical results have been calculated using eq. (19), (28), (34), (36), and (43). 
i Parallel lines pointing to distant observer

I

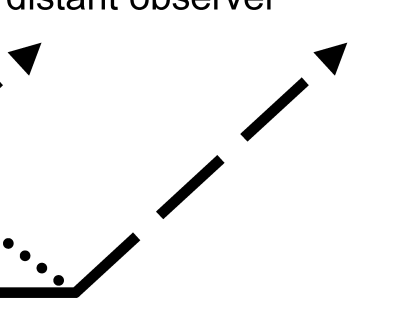

Source 1

Source 2 


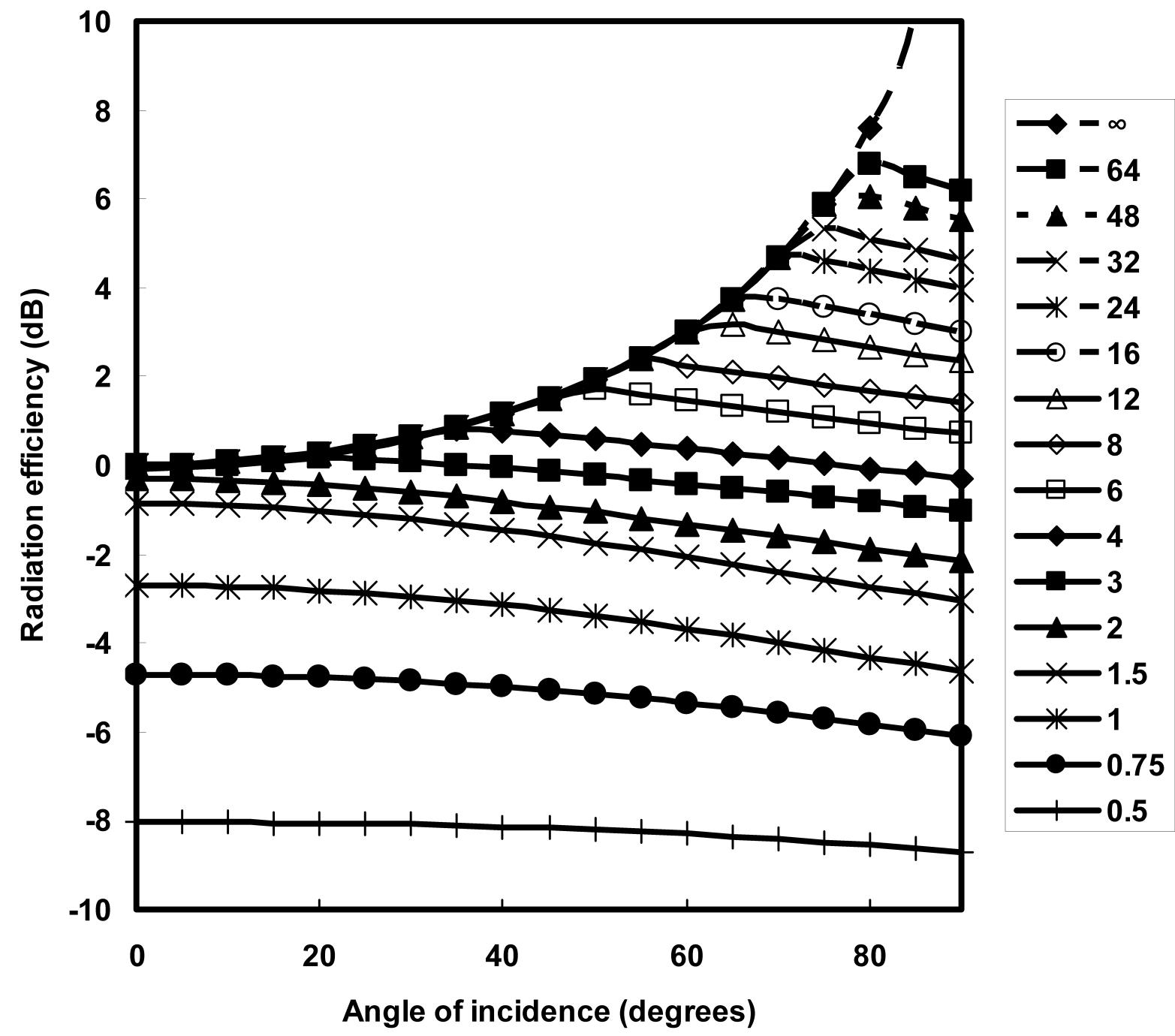




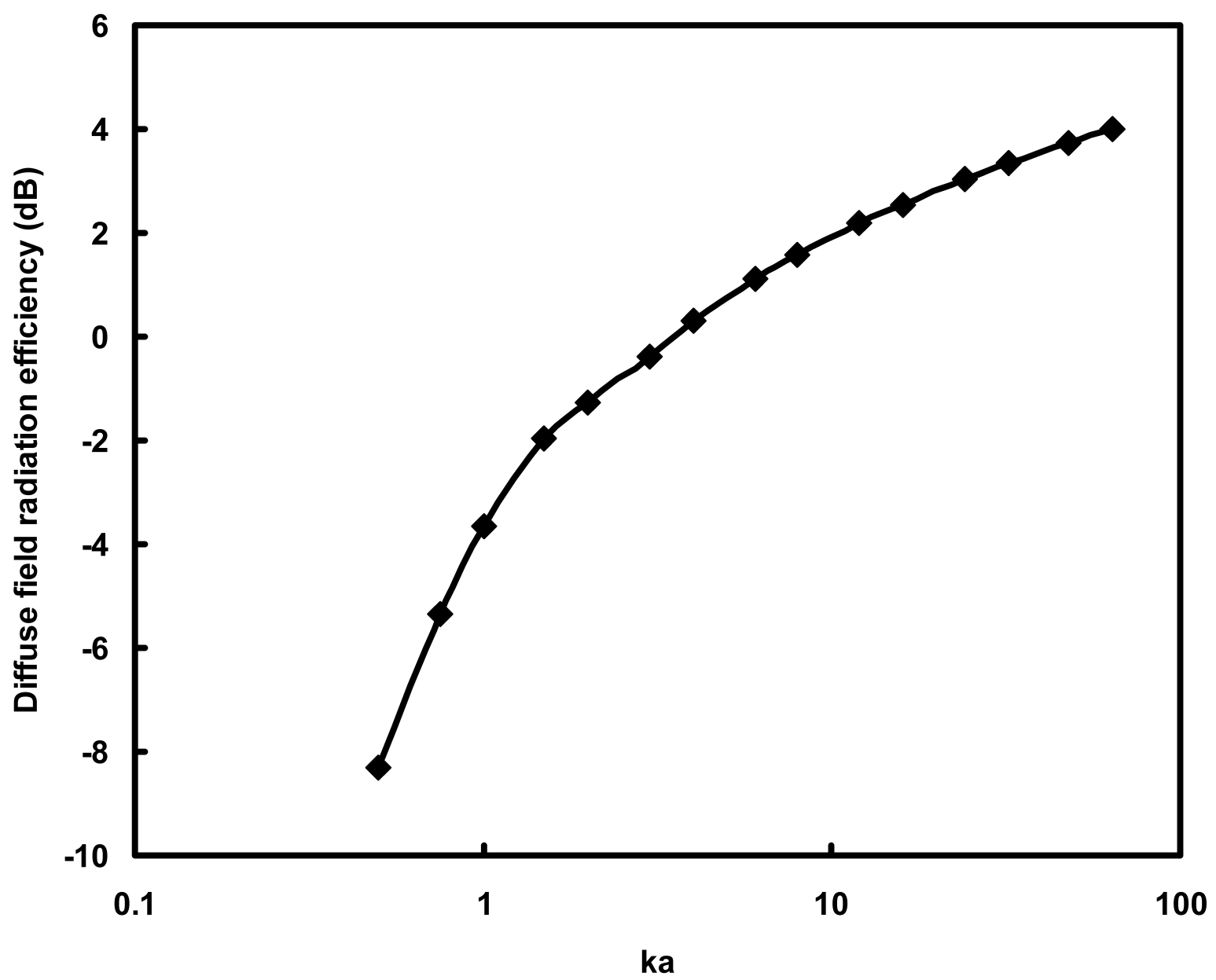

